\title{
Perfil psicológico de atletas brasileiros de Paradesporto em Nível Escolar
}

Psychological profile of brazilian athletes of Paradesports at School Level

Perfil psicológico de los Paradeportistas brasileños a Nivel Escolar

\section{Resumo}

A prática esportiva por pessoas com deficiência vem crescendo cada vez mais, trazendo consigo a necessidade de maiores investimentos em diversos segmentos, como por exemplo, a investigação científica. Buscar compreender os fenômenos que envolvem os paratletas em seu universo esportivo torna-se relevante em função das dimensões psicológicas envolvidas, sabendo que são atletas que se preparam para o alto rendimento. A presente pesquisa teve como objetivo caracterizar os perfis psicológicos predominantes relacionados à motivação e resiliência de atletas brasileiros de paradesporto em nível escolar, além de verificar possíveis associações entre o perfil psicológico e fatores sócio-demográficos e de formação esportiva, assim como, analisar as correlações entre as dimensões exploradas. Tratou-se de uma pesquisa quantitativa de caráter descritivo. Participaram 114 atletas paralímpicos em nível escolar, de escolas públicas e privadas brasileiras, de ambos os sexos $(\mathrm{F}=21,9 \% ; \mathrm{M}=78,1 \%)$, com idade média de 15,45 anos $( \pm 1,44)$, com deficiência física e visual, de 21 estados brasileiros, praticantes de variadas modalidades paralímpicas. Os participantes responderam a instrumentos validados de motivação e de resiliência, além de uma anamnese com o intuito de caracterizá-los. Foi possível constatar que a amostra se dividiu em dois grandes grupos de perfis, o primeiro com valores altos de motivação e resiliência, e o segundo com valores moderados destas mesmas variáveis. Ao analisar características sócio-demográficas e de formação esportiva, observou-se que somente a deficiência e a idade tiveram valores significativos de interferência nesta divisão dos sujeitos.

Palavras-chave: Resiliência; Motivação; Perfil; Paradesporto; Escolar.

\begin{abstract}
The practice of sports by people with disabilities has been growing more and more, bringing with it the need for greater investments in several segments, such as, for example, scientific research. Seeking to understand the phenomena that involve the athletes in their sports universe becomes relevant due to the psychological dimensions involved, knowing that they are athletes who prepare for high performance. The present research aimed to characterize the predominant psychological profiles related to the motivation and resilience of Brazilian sportspeople at school level, in addition to verifying possible associations between the psychological profile and sociodemographic and sports training factors, as well as analyzing the correlations between the dimensions explored. It was a quantitative research of a descriptive character. 114 Paralympic athletes participated at school level, from public and private Brazilian schools, of both sexes $(\mathrm{F}=21.9 \%$; $\mathrm{M}=78.1 \%)$, with an average age of 15.45 years $( \pm 1.44)$, with physical and visual disabilities, from 21 Brazilian states, practicing various Paralympic modalities. Participants responded to validated instruments of motivation and resilience, in addition to an anamnesis in order to characterize them. It was possible to verify that the sample was divided into two large groups of profiles, the first with high values of motivation and resilience, and the second with moderate values of these same variables. When analyzing sociodemographic and sports training characteristics, it was observed that only disability and age had significant values of interference in this division of the subjects.
\end{abstract}

Keywords: Resilience; Motivation; Profile; Paradeport; Schooler. 


\begin{abstract}
Resumen
La práctica de deporte por parte de las personas con discapacidad ha crecido cada vez más, trayendo la necesidad de mayores inversiones en varios segmentos, como por ejemplo, la investigación científica. Buscando comprender los fenómenos que involucran a los deportistas en su universo deportivo, cobra relevancia por las dimensiones psicológicas involucradas, sabiendo que son deportistas que se preparan para el rendimiento. Esta investigación tuvo como objetivo caracterizar los perfiles psicológicos predominantes relacionados con la motivación y resiliência de los deportistas brasileños a nivel escolar, además de verificar las posibles asociaciones entre el perfil psicológico y los factores sociodemográficos y de entrenamiento deportivo, así como analizar las correlaciones entre las dimensiones exploradas. Fue una investigación cuantitativa de carácter descriptivo. Participaron 114 atletas paralímpicos en nivel de la escuela, de escuelas brasileñas públicas y privadas, de ambos los géneros $(\mathrm{M}=21,9 \% ; \mathrm{H}=78,1 \%)$, con edad promedio de 15,45 años $( \pm 1,44)$, con discapacidad física y visual, de 21 provincias brasileñas, practicantes de distintas modalidades paralímpicas. Los participantes respondieron a instrumentos validados de motivación y de resiliencia, además de una anamnesis con el fin de caracterizar la muestra. Así, se pudo comprobar que la muestra se dividió en dos grandes grupos de perfiles, el primero con valores altos de motivación y resiliencia, y el segundo con valores moderados de estas mismas variables. Al analizar las características sociodemográficas y del entrenamiento deportivo, se observó que solo la discapacidad y la edad tenían valores significativos de interferencia en esta división de los sujetos.
\end{abstract}

Palabras clave: Resiliencia; Motivación; Perfil; Paradeporto; Escolar.

\title{
1. Introdução
}

A prática de exercícios e esportes para pessoas com deficiência, bem como do Esporte Paralímpico, no âmbito nacional e internacional, vêm crescendo significativamente nas últimas décadas. Assim, consquentemente, todo o cenário envolto à esse segmento, também acompanha esse movimento e vêm evoluindo e se aprimorando ao longo desses anos (Matos, Cruz \& Almeida, 2011; Samulski, 2009). Tratando-se desse crescimento no território nacional, é perceptível uma grande popularização de tais práticas, após o Brasil ter sediado os Jogos Paralimpicos, no Rio de Janeiro, no ano de 2016, como destacado na época, pelo presidente do Comitê Paralímpico Brasileiro, Andrew Parsons (Parsons, 2016).

Como afirmado, com esse crescimento, houve a necessidade de outras características do esporte se desenvolverem, considerando principalmente, aspectos físicos dos atletas, aspectos sociais e por fim, os psicológicos. Ressaltando que este último, será o foco deste estudo, visto que as questões psicológicas geram impactos decisivos e são consideradas atualmente como fator diferencial em atletas, e por isso, devem ser estudadas, controladas e utilizadas de forma a beneficiar o atleta ou praticante (Bodas, Lázaro \& Fernandes, 2004; Levermore, 2008; Sylta et al., 2016).

Por esse motivo, muitos autores destacam a importância do controle das esferas psicológicas na busca de melhores resultados e desempenho, aliado à preparação física, tática e técnica desses atletas. Sendo assim, identificar perfis psicológicos desses sujeitos, pode auxiliar dentro dos treinamentos, competições e até em suas vidas pessoais (Bodas, Lázaro, \& Fernandes, 2004; Silva, 2016; Samulski, 2009; Samulski \& Noce, 2002; Weinberg \& Gould, 2008). Isto se torna ainda mais relevante, considerando atletas em nível escolar, que almejam e estão se preparando, em alguns casos, para o período de transição no esporte, rumo ao profissionalismo.

Ao longo dos anos, estudiosos buscaram traçar o perfil psicológico de atletas com e sem deficiência, para isso, realizaram diversos estudos partindo de pontos de vista distintos (Samulski \& Noce, 2002; Bodas, Lázaro \& Fernandes, 2007; Cusin \& Navarro, 2013; Santos, Ribeiro \& Carvalho, 2014). Segundo João \& Fernandes Filho (2002), o perfil pode incluir informações sobre características psicológicas, genéticas e/ou somatotípicas de determinado indivíduo ou grupo. No meio esportivo, a partir do conhecimento de tais características, profissionais conseguem determinar a aptidão de determinado atleta para uma modalidade específica, em que seja compatível com seu perfil. Soares \& Machado (2004) complementam, afirmando que a definição destas características auxilia na escolha de estratégias mais apropriadas para cada atleta. Outros autores ainda concordam com os supracitados, alegando que a partir do conhecimento destes perfis, obtêm-se formas de trabalhar e de agir de forma mais eficiente, resultando em um melhor rendimento de seus atletas ou grupo (Medina \& Fernandes Filho, 2002). 
Inicialmente, pode-se destacar um estudo de Samulski \& Noce (2002), que avaliou 64 atletas de oito esportes distintos em busca da definição do perfil psicológico desses sujeitos. Para isto, foi aplicado: testes de personalidade, motivação, estresse e testes psicométricos, como o de percepção, tempo de reação e concentração. Observou-se que houve diferença significativa em relação aos tipos de deficiência e aos sexos. E os motivos mais importantes para inserção às atividades esportivas, foram, o prazer e a busca pela reabilitação; e os principais para praticar esportes, a competitividade e superação. Em relação aos fatores estressantes, pode-se destacar com maior frequência, os problemas com sono, pressão e os conflitos interpessoais. Quanto aos testes psicométricos, a maioria dos atletas apresentou bons resultados de tempo de reação e percepção.

Já outro estudo, procurou definir o perfil psicológico de atletas portugueses que participaram das Paralimpíadas de Atenas, em 2004. A amostra foi composta por 28 atletas de quatro modalidades e três tipos de deficiência, que responderam a um questionário que avaliava sete principais variáveis, autoconfiança; pensamentos negativos; pensamentos positivos; atenção; visualização; motivação e atitude competitiva. Como resultado, percebeu-se que a motivação fora o aspecto com níveis mais elevados, seguido da autoconfiança e atitude competitiva. Fatores como o tipo de deficiência, modalidade e títulos conquistados também interferiram de forma significativa nos resultados (Bodas \& Lázaro, 2007).

Em uma terceira pesquisa, conduzida por Cusin \& Navarro (2013), foi investigado o perfil de atletas de futsal feminino. 110 atletas de equipes participantes da Federação Paulista de 2012 responderam a um questionário que avaliava 15 características psicológicas. De forma geral, a variável com valor mais elevado foi a Resiliência (80,4\%), seguida da Competitividade (79,0\%), Coletividade (78,9\%), Autoconfiança (77,6\%), Persistência (77,0\%), Comprometimento (76,2\%), Disciplina (75,5\%), Estratégia (74,9\%), Incentivo (73,6\%), Autoconhecimento e Concentração (73,3\%), Dinamismo (71,6\%), e por fim, Comunicação $(63,6 \%)$ e Liderança $(61,9 \%)$. Acreditando que tais resultados, contribuam de forma positiva na formulação de treinos mais adequados, em busca de melhorias no desempenho, nas relações interpessoais e no desenvolvimento de características de cada atleta.

E por fim, ainda em busca de analisar o perfil psicológico, uma investigação foi realizada por Santos, Ribeiro \& Carvalho (2014), com atletas de voleibol, de 18 a 21 anos. Para isso, aplicou-se uma adaptação de dois testes denominados Test de las presiones del deportista de Selección e Test de los miedos del futbolista. Os resultados obtidos apresentaram o medo de fatores como lesões, o medo do futuro e a forte auto-exigência por parte dos atletas. Os autores consideram que estes fatores estressores são em virtude de elementos extrínsecos e intrínsecos, exigindo e aumentando a importância da participação e acompanhamento de um psicólogo esportivo na preparação da equipe e de cada atleta individualmente.

Como observado acima, pesquisadores partem da investigação de variáveis distintas para se traçar perfis, neste caso especialmente, buscou-se analisar o perfil psicológico a partir da motivação e capacidade de resiliência dos sujeitos. Como afirmado por Samulski (2009), a motivação depende de fatores internos (pessoais) e externos (ambientais). Dentro desse contexto, existem diversos fatores que influenciam o rendimento do atleta, dentre eles, um dos principais é a motivação, seguido de autoestima, autoconfiança, ansiedade e agressividade. Esta variável é considerada o objeto de estudo mais importante na psicologia esportiva, na busca de conhecimento acerca de sua influência nos treinamentos e competições. Além disso, pode ser considerada como o motor do esporte, responsável por explicar as razões de inserção, manutenção e abandono da prática, podendo ser determinada e sofrer influências internas e externas (Pujals \& Vieira, 2002; Freitas et al., 2007; Dosil, 2004).

Já a resiliência, define-se como a capacidade de vivenciar experiências em adversidades sucessivas sem possíveis prejuízos em seu desenvolvimento, prevalecendo a superação e fortalecimento diante das adversidades (Grunspun, 2006). Aplicando este conceito no esporte, o atleta deve superar diversas dificuldades ao longo de sua carreira e de sua formação esportiva, transformando tudo em experiências que possam fortalecê-lo e motivá-lo. Muitas características vinculadas ao sucesso do atleta, como por exemplo, a auto-eficácia, autoconfiança, orientação à meta, otimismo, minimização do stress, foco, 
compromisso, estão vinculados a esse conceito. A atividade esportiva pode favorecer o praticante, ao enfrentar as situações adversas no contexto esportivo, e que assim, ele seja capaz de transpor essa vivência para outros campos de sua vida, possibilitando uma melhor preparação e confiança para enfrentar as dificuldades (Milham, 2007; Sanches \& Rubio, 2010).

Além desta capacidade de transferir questões resilientes do meio esportivo para a vida, Gonçalves et al., (2006) enaltecem que o esporte também é responsável por contribuir em outros aspectos, como na formação de caráter, respeito ao próximo, disciplina, dentre outros. Ademais, para Garcia, Medeiro e Zaremba (2011), a prática esportiva é um espaço no qual se aprende lições e valores, contribuindo para o desenvolvimento psicossocial, favorecendo a formação destes sujeitos e tornando-os mais empenhados, batalhadores, honestos, éticos e comprometidos. Reforçando que a prática esportiva é benéfica fisicamente, psicologicamente e socialmente para crianças e jovens (Silva \& Costa Jr., 2011).

Sendo assim, este estudo objetivou definir os perfis psicológicos predominantes relacionados à motivação e a resiliência de atletas brasileiros de paradesporto em nível escolar, além de verificar as possíveis associações entre os perfis psicológicos identificados, os fatores sócio-demográficos e de formação esportiva, além de também analisar as correlações existentes entre as dimensões analisadas.

\section{Metodologia}

\section{Tipo de Pesquisa}

Trata-se de uma pesquisa quantitativa de caráter descritivo. Segundo Fonseca (2002), a pesquisa quantitativa, possui por objetivo quantificar com objetividade. Além disso, afirma que este tipo de estudo recorre a uma linguagem matemática para descrever causas de um fenômeno e relações entre variáveis. Tem como característica a utilização de instrumentos padronizados.

Para Figueiredo (2008) e Gil (2008), estudos de caráter descritivo buscam primordialmente, descrever características de determinado fato, fenômeno ou população, e pode estabelecer relações entre variáveis. Gil (2008) é mais específico, ao afirmar que esse tipo de estudo busca investigar as características de um grupo, como por exemplo, sua distribuição quanto a idades, procedência, nível escolar, sexo, estado de saúde física ou mental, dentre muitas outras variáveis.

\section{Aspectos Éticos}

O desenvolvimento desta pesquisa obteve o consentimento dos responsáveis legais pelas instituições e dos próprios participantes e de seus responsáveis, após aprovação pela Comissão de Ética em Pesquisa Envolvendo Seres Humanos da Universidade Federal de São João Del Rei. Este estudo deriva-se de um projeto maior denominado "Promoção de Saúde e suas Interfaces Físicas e Psicológicas: programa de intervenção e pesquisa com idosos, pessoas com deficiência, adultos com diabetes e hipertensos" sob o número 032/2013.

\section{Participantes da Pesquisa}

Participaram do estudo 114 atletas em nível escolar, participantes da maior competição paralímpica nacional estudantil, praticantes de Atletismo, Futebol de 7, Goalball, Judô, Natação, Tênis de Mesa e Tênis em Cadeira de Rodas. Possuíam deficiência física $(71,9 \%)$ e visual $(28,1 \%)$, (não fazendo parte do estudo as pessoas com deficiência intelectual, já que os instrumentos adotados para coleta de dados foram questionários validados, garantindo a validade dos resultados), de ambos os sexos $(\mathrm{F}=21,9 \% ; \mathrm{M}=78,1 \%)$, com idades variadas entre 12 a 17 anos $(15,45 \pm 1,44)$, provenientes de escolas públicas (86\%) e privadas (14\%). O critério para escolha dos participantes foi por conveniência, de acordo com a disponibilidade e consentimento dos atletas e dos técnicos durante o evento, ocorrido em São Paulo, no ano de 2017. 
A amostra abrangeu atletas de quase todo o território brasileiro, representando 20 estados, mais o Distrito Federal, de todas as cinco regiões do Brasil. Os estados com número maior de respondentes são São Paulo (15,8\%), Santa Catarina (14,9\%), seguido de Mato Grosso do Sul (10,5\%) e Rio de Janeiro (9,6\%), com participações menores apresentou-se Paraná, Rondônia e Tocantins com apenas $0,9 \%$ cada estado. Na Figura 1, é apresentado um mapa detalhado sobre a distribuição dos sujeitos por estados e regiões.

Figura 1 - Participantes da pesquisa de acordo com os estados e regiões Brasileiras.

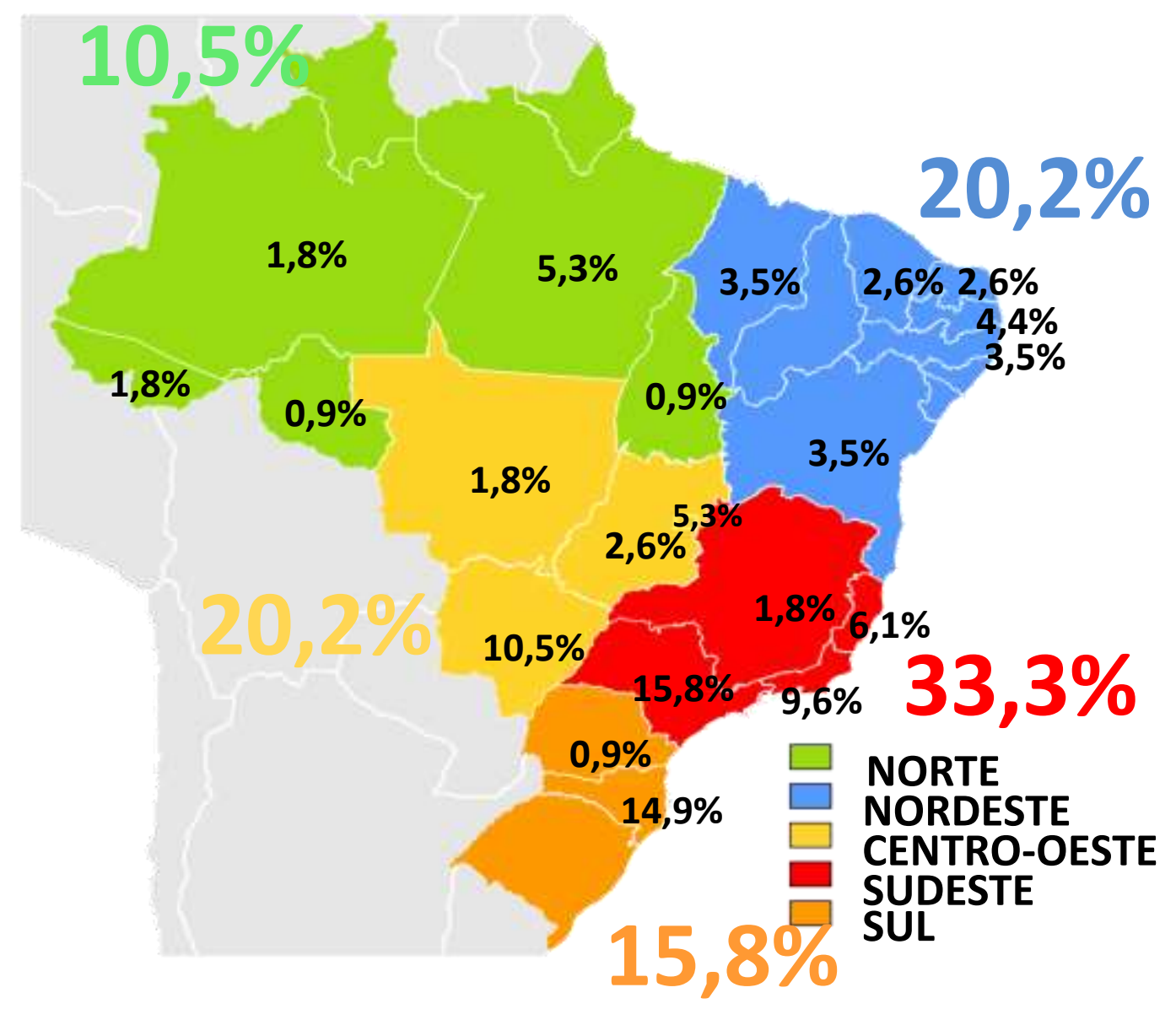

Fonte: Coleta de Dados (2017).

\section{Instrumentos}

Anamnese

Breve anamnese composta por questões relacionadas aos atletas, buscando caracterizá-los sócio-demograficamente e esportivamente.

Inventário de Motivação à Prática Regular de Atividade Física (IMPRAF-54).

É uma versão reduzida de sua original, validado e proposto por Balbinotti \& Barbosa (2006). Composta por 54 itens, divididos em seis categorias, Controle de Estresse $(\alpha=0,89)$, Saúde $(\alpha=0,87)$, Sociabilidade $(\alpha=0,94)$, Competitividade $(\alpha=$ 0,94), Estética $(\alpha=0,91)$ e Prazer $(\alpha=0,82)$. As respostas são dadas conforme uma escala de tipo Likert, de 1 a 5 pontos, partindo de “isto me motiva pouquíssimo" (1) até “isto me motiva muitíssimo” (5). Cada dimensão é analisada 
individualmente, para isso, é necessário fazer a somatória das questões que a compõem para se obter os escores brutos de cada uma.

No que se refere à motivação, o instrumento a divide em seis principais dimensões: "Controle de Estresse", que expressa o desejo do sujeito de obter alívio de ansiedade, angústias, irritação e estresse, a partir da prática esportiva; "Saúde", relacionada ao interesse nos possíveis benefícios à saúde que o exercício físico pode trazer; "Sociabilidade", referente a oportunidade de criação de vínculos sociais, reunir, estar ou se encontrar com amigos; "Competitividade", que exprime o sentimento de querer competir, concorrer e ganhar dos outros; "Estética", que revela o desejo de ter ou ficar com um corpo bonito e definido; e finalmente, "Prazer", que mede a satisfação, o bem estar e a auto realização a partir da prática esportiva (Balbinotti \& Barbosa, 2006).

\section{Escala de Resiliência}

Considerado um dos poucos instrumentos para medir níveis de resiliência, foi desenvolvida por Wagnild \& Young (1993) e sua adaptação transcultural foi proposta por Herdman, Fox-Rushby \& Badia (1998) e divulgada por Hasselmann \& Reichenheim (2003) e Moraes, Hasselmann \& Reichenheim, (2002) na versão da língua portuguesa. É composta por 25 itens, distribuídos em cinco dimensões propostas pelo modelo ER5: Auto-suficiência $(\alpha=0,69)$; Sentido de Vida $(\alpha=0,70)$; Equanimidade $(\alpha=0,75)$; Perseverança $(\alpha=0,79)$; e Singularidade Existencial $(\alpha=0,56)$ (Perin, Dias, Corte-Real, Andrade $\&$ Fonseca, 2015). As resposta são realizadas através de uma escala tipo Likert de 7 pontos, desde "Discordo fortemente" até "Concordo fortemente". Os escores podem variar de 25 a 175 pontos, com valores altos indicando elevada capacidade de resiliência (acima de 145 pontos), médios, representando resiliência média (125-145 pontos) e os menores, indicando baixa resiliência (até 125 pontos) (Wagnild \& Young, 1993).

Para esta pesquisa, utilizou-se um modelo baseado no original citado acima, no qual, os respondentes avaliaram seus níveis em uma escala tipo Likert de 1 a 4, onde 1 representa "Discordo fortemente" e 4 expressa "Concordo fortemente", com as pontuações finais modificadas de acordo com esse novo modelo: valores abaixo de 71, representam baixa resiliência, de 72 a 83, com resiliência média, e superior a 84, como alta resiliência.

Como supracitado, a resiliência, a partir do instrumento utilizado, pode ser dividida em cinco dimensões, a primeira delas denomina-se "Auto-Suficiência", que se relaciona à autoconfiança do sujeito, ou seja, ao quanto ele confia em suas potencialidades e sobre o autoconhecimento de seus limites; a segunda, "Sentido de Vida", pode ser definida como a percepção do sujeito de que a sua vida possui um sentido, um propósito, crença nos bons motivos existentes para se viver; a terceira, chamada "Equanimidade" refere-se à capacidade de encarar diversos acontecimentos da vida com flexibilidade e boa aceitação; a "Perseverança", expressa a capacidade de continuar a seguir em frente, apesar das adversidades encontradas pelo caminho; e por fim, a "Singularidade Existencial" representa o sentimento de singularidade do sujeito, ou seja, de ser único (Wagnild \& Young, 1993).

\section{Procedimento de Coleta de Dados}

$\mathrm{O}$ procedimento da coleta de dados pautou-se nos seguintes processos: a) Inicialmente, enviou-se à Academia Paralímpica Brasileira (APB) e ao Comitê Paralímpico Brasileiro (CPB), o projeto de pesquisa para prévia autorização para a realização do estudo em uma competição oficial em nível escolar, seguindo todos os parâmetros do Comitê de Ética da Universidade Federal de São João Del Rei, nº 466 de 2012; b) Posteriormente, após autorizada a pesquisa, já no local e nos dias do evento esportivo, a pesquisadora entrou em contato com diversos treinadores (responsáveis legais pelos atletas menores de idade em momento de competição), explicando-lhes a pesquisa, bem como seus procedimentos, e pedindo-lhes permissão de convite aos seus atletas; c) Após isto, os treinadores que concordaram e autorizaram a participação de seus atletas, aceitaram 
e assinaram o Termo de Consentimento Livre e Esclarecido (TCLE), autorizando detalhadamente determinados atletas à participarem da pesquisa e direcionando-os à pesquisadora; d) Já em contato com os atletas autorizados, foi explicada mais uma vez, a pesquisa e seus procedimentos e lido o Termo de Assentimento Livre e Esclarecido (TALE), de forma clara e simples, em uma linguagem adequada e de fácil acesso à eles, e assim, aqueles que concordaram em participar, assinaram ao TALE; e) Por fim, estes sujeitos foram direcionados a responderem uma breve anamnese, com o intuito de caracterizá-los sócio-demograficamente e em relação às suas formações esportivas, e finalmente, responderam aos instrumentos: Inventário de Motivação à Prática Regular de Atividade Física e à Escala de Resiliência.

\section{Análise Estatística}

A metodologia de análise dos dados foi realizada através do software de fomentação de planilhas e procedimentos estatísticos, denominado Software Statistical Package for the Social Sciences (SPSS) versão 20.0.

Primeiramente, à parte deste processo, seguiu-se as orientações próprias de cada instrumento, podendo-se extrair valores totais de cada variável (motivação e resiliência) e também de suas dimensões. Posteriormente, utilizou-se uma análise descritiva, com valores de frequência, média, porcentagens e desvio padrão.

Quanto às análises estatísticas mais aprofundadas, em um primeiro momento, separou-se a amostra em clusters (grupos), onde se definiu dois grandes grupos, eliminando apenas dois sujeitos da amostra total, considerados outliers.

O objetivo dessa análise foi verificar, também, se os atletas poderiam ser agrupados segundo perfis psicológicos predominantes, considerando os construtos de motivação e resiliência. Para isso, foi utilizada a Análise de Cluster ou Agrupamento, buscando agrupar os participantes por similaridade (Hair et al., 2005). Foi utilizada a técnica Hierárquica Aglomerativa e o método utilizado foi o de Ward, baseado nos princípios da análise de variância. A medida de similaridade utilizada foi a distância euclidiana, que representa a similaridade como a proximidade entre observações ao longo das variáveis na variável estatística de agrupamento. Para determinar o número de conglomerados, utilizou-se o gráfico dos passos de agrupamentos em relação ao nível de distância, sendo identificados dois conglomerados: Alta motivação e resiliência (Cluster 1) e Moderada motivação e resiliência (Cluster 2). Por último, foi realizada a Análise de Variância (ANOVA), para verificar se havia diferenças entre as médias dos dois agrupamentos.

Após este procedimento, recorreu-se ao Teste de Qui-quadrado para investigar quais variáveis, dentre as sóciodemográficas e de formação esportiva, dependeriam a distribuição dos sujeitos dentre os clusters, e por fim, utilizou-se o Coeficiente de Pearson para analisar o nível de relação entre as dimensões dos instrumentos de Motivação e Resiliência.

\section{Resultados e Discussão}

A tabela a seguir, caracteriza sócio-demograficamente e em relação à formação esportiva os participantes da pesquisa: 
Research, Society and Development, v. 10, n. 4, e17310414044, 2021

(CC BY 4.0) | ISSN 2525-3409 | DOI: http://dx.doi.org/10.33448/rsd-v10i4.14044

Tabela 1 - Características sócio-demográficas e de formação esportiva dos participantes do estudo.

\begin{tabular}{|c|c|c|c|}
\hline Variável & Descrição & $\mathbf{F}$ & $\mathbf{P}(\%)$ \\
\hline \multirow{2}{*}{ Sexo } & Masculino & 89 & $78,1 \%$ \\
\hline & Feminino & 25 & $21,9 \%$ \\
\hline \multirow{2}{*}{ Deficiência } & Física & 82 & $71,9 \%$ \\
\hline & Visual & 32 & $28,1 \%$ \\
\hline \multirow{2}{*}{$\begin{array}{c}\text { Tipo de } \\
\text { deficiência }\end{array}$} & Congênita & 95 & $83,3 \%$ \\
\hline & Adquirida & 19 & $16,7 \%$ \\
\hline \multirow{2}{*}{ Escola } & Pública & 98 & $86 \%$ \\
\hline & Privada & 16 & $14 \%$ \\
\hline \multirow{7}{*}{ Modalidade } & Atletismo & 31 & $27,2 \%$ \\
\hline & Futebol de 7 & 22 & $19,3 \%$ \\
\hline & Goalball & 13 & $11,4 \%$ \\
\hline & Judô & 9 & $7,9 \%$ \\
\hline & Natação & 24 & $21,1 \%$ \\
\hline & Tênis de Mesa & 8 & $7 \%$ \\
\hline & Tênis em Cadeira de Rodas & 7 & $6,1 \%$ \\
\hline \multirow{5}{*}{ Região } & Norte & 12 & $10,5 \%$ \\
\hline & Sul & 18 & $15,8 \%$ \\
\hline & Centro-Oeste & 23 & $20,2 \%$ \\
\hline & Nordeste & 23 & $20,2 \%$ \\
\hline & Sudeste & 38 & $33,3 \%$ \\
\hline \multirow{6}{*}{ Idade } & 12 & 6 & $5,3 \%$ \\
\hline & 13 & 6 & $5,3 \%$ \\
\hline & 14 & 15 & $13,2 \%$ \\
\hline & 15 & 26 & $22,8 \%$ \\
\hline & 16 & 26 & $22,8 \%$ \\
\hline & 17 & 35 & $30,7 \%$ \\
\hline \multirow{7}{*}{$\begin{array}{c}\text { Tempo } \\
\text { De } \\
\text { Prática }\end{array}$} & Até 1 ano & 31 & $27,2 \%$ \\
\hline & 2 anos & 16 & $14 \%$ \\
\hline & 3 anos & 14 & $12,3 \%$ \\
\hline & 4 anos & 15 & $13,2 \%$ \\
\hline & 5 anos & 15 & $13,2 \%$ \\
\hline & 6 anos & 6 & $5,3 \%$ \\
\hline & 7 anos ou mais & 17 & $14,9 \%$ \\
\hline \multirow{3}{*}{$\begin{array}{c}\text { Carga } \\
\text { Horária } \\
\text { Semanal }\end{array}$} & Até 4 horas & 53 & $46,5 \%$ \\
\hline & $5 \mathrm{~h}-8 \mathrm{~h}$ & 33 & $28,9 \%$ \\
\hline & Acima de 9h & 28 & $24,6 \%$ \\
\hline
\end{tabular}

Legenda da Tabela 1: F = Frequência; $\mathrm{P}=$ Porcentagem. Fonte: Autores (2017).

Ao analisar os participantes da pesquisa quanto às idades, a maior participação concentrou-se em atletas com 17 anos, o limite para participação na competição escolar em questão, representando 30,7\% da população estudada, com 15 e 16 anos, $22,8 \%$ cada e de 12 e 13 anos, simbolizando 5,3\% cada.

Ao que se refere aos sexos, há predominância de sujeitos do sexo masculino $(78,1 \%)$, enquanto as do sexo feminino representam apenas $21,9 \%$ dos integrantes. Somente $14 \%$ deles, são provenientes de escolas privadas, e a grande maioria, $86 \%$, de escolas públicas. 
Em relação à deficiência, $71,9 \%$ dos componentes possuem comprometimento físico, e os outros $28,1 \%$, visual. Quanto ao tipo de deficiência, $83,3 \%$ apresentam uma deficiência congênita, ou seja, já nasceram com ela, e $16,7 \%$ a adquiriram de alguma forma.

Quanto às modalidades esportivas, a maior parte dos participantes são atletas de atletismo $(27,2 \%)$, seguidos de natação $(21,1 \%)$, futebol de 7 (19,3\%), goalball $(11,4 \%)$, judô $(7,9 \%)$, tênis de mesa (7\%), e por fim, tênis em cadeira de rodas, representando apenas $6,1 \%$ da amostra.

Sobre as regiões, o Sudeste apresenta-se como a região com mais respondentes da pesquisa, com 33,3\% e subsequentes o Centro-Oeste e Nordeste (ambas com 20,2\%), posteriormente Sul $(15,8 \%)$ e Norte $(10,5 \%)$.

Por fim, as características relacionadas à formação esportiva, o tempo de prática dos atletas varia de 1 mês até 168 meses (14 anos), e a carga horária semanal de treinamento, de 1 hora até 30 horas/semanais. A maior frequência no tempo de prática, foi de até 1 ano (27,2\%), e em relação a carga horária, até 4 horas semanais, representando 46,5\%.

Na Tabela 2, pode-se observar a divisão da amostra em dois grandes grupos. A partir de uma análise de perfis, a amostra foi subdividida em dois subgrupos, o primeiro denominado Cluster 1 (Alta motivação e resiliência) e o segundo, como Cluster 2 (Moderada motivação e resiliência):

Tabela 2 - Clusters de Motivação e Resiliência.

\begin{tabular}{ccccc}
\hline Cluster/ Fatores & $\begin{array}{c}\text { Cluster 1 } \\
(\mathbf{n}=\mathbf{5 3})\end{array}$ & $\begin{array}{c}\text { Cluster 2 } \\
(\mathbf{n = 5 9})\end{array}$ & $\begin{array}{c}\text { F } \\
\text { (entre grupos) }\end{array}$ & Sig \\
\hline Resiliência Geral & 84,38 & 78,46 & 17,628 & $0,000^{*}$ \\
Auto-Suficiência & 16,77 & 15,34 & 8,311 & $0,005^{*}$ \\
Sentido de Vida & 17,58 & 16,71 & 6,144 & $0,015^{*}$ \\
Equanimidade & 15,21 & 13,86 & 9,865 & $0,002^{*}$ \\
Perseverança & 17,74 & 16,68 & 7,113 & $0,009^{*}$ \\
Singularidade Existencial & 17,08 & 15,86 & 4,769 & $0,031^{*}$ \\
Motivação Geral & 245,47 & 195,14 & 241,900 & $0,000^{*}$ \\
Controle de Estresse & 36,19 & 27,08 & 43,403 & $0,000^{*}$ \\
Saúde & 43,43 & 36,02 & 79,769 & $0,000^{*}$ \\
Sociabilidade & 41,17 & 34,12 & 30,912 & $0,000^{*}$ \\
Competitividade & 42,13 & 32,57 & 57,009 & $0,000^{*}$ \\
Estética & 39,60 & 26,71 & 86,210 & $0,000^{*}$ \\
Prazer & 42,94 & 38,24 & 37,756 & $0,000^{*}$ \\
\hline
\end{tabular}

*Significante $<0,05$ (2-tailed). Fonte: Das autoras (2017).

Como visto acima, o Cluster 1, foi composto por 53 indivíduos e o Cluster 2, por 59 sujeitos, totalizando 112 atletas, os dois atletas restantes da amostra, foram desconsiderados nesta separação, já que possuíam valores que não se encaixavam na dinâmica de divisão, ou seja, foram considerados sujeitos outliers. Analisando os valores descritos na Tabela 2, o Cluster 1 é considerado o mais motivado e resiliente para a prática esportiva, enquanto o Cluster 2, de motivação e resiliência moderadas para tal prática.

Altos níveis de motivação, podem consequentemente provocar modificações no desempenho e rendimento de atletas, assim como, as experiências no esporte podem contribuir para o aumento da resiliência, e um perfil mais resiliente pode estar associado a um melhor desempenho no esporte (Almeida, 2009; Cevada et al., 2012).

Outros autores complementam, afirmando que o envolvimento de pessoas com deficiência nas práticas esportivas, favorece o desenvolvimento da resiliência. Assim, atletas ou praticantes de exercícios que apresentem índices ideais de resiliência, conseguem enfrentar situações adversas no esporte, como pressões, imprevisibilidades, desafios e dificuldades, de maneira mais saudável, mantendo a constante motivação para realizar as tarefas da melhor maneira possível. Deste modo, fica 
clara a relação entre resiliência e motivação para obtenção de bons resultados (Veloso, Celani \& Scheeren, 2018; Rice et al., 2016; Wagstaff et al., 2016).

Mais especificamente com atletas paralímpicos, Codonhato et al., (2018), Martin \& Wheeler (2011) e Brown, But \& Sarkar, (2019) destacam que, atletas com deficiência lidam com fatores estressores, rotina de treinamentos, competição, acessibilidade ou a falta dela e problemas pessoais, como oportunidades de crescimento e vivenciam isto como desafios a serem superados, dia a dia, e por isso, muitos deles utilizam o desporto para desenvolver seus mecanismos de resiliência. Nesse sentido, entende-se que lidar com a deficiência, em determinado momento da vida, será sempre uma oportunidade de desenvolver a resiliência e a necessidade de constantes readaptações. Solano et al., (2016) e Brown, But \& Sarkar (2019), ainda salientam que, a tolerância e a capacidade de adaptação estão intimamente ligadas à recuperação diante de adversidades.

Já no segmento motivacional, Cavalcante (2011) acredita que esta variável seja a força impulsiva para que as pessoas atinjam seus objetivos, mantendo um comportamento linear e adequado para isso. Tratando-se do esporte, isso fica ainda mais claro, levando em consideração a construção de uma carreira esportiva para atingir o alto rendimento. Para isso, Silva (2019) destaca os fatores intrínsecos de um atleta e também os extrínsecos, como decisivos para alcançar bons resultados.

Busto (2013) complementa, afirmando que as práticas paradesportivas, também são responsáveis pelo surgimento de sensação de bem estar, menores níveis de ansiedade e depressão e na melhora de aspectos psicológicos, como a motivação. Isso justifica o fato de os sujeitos da presente pesquisa, apresentarem níveis medianos e ótimos de motivação, e não se caracterizarem como grupos desmotivados.

Já na Tabela 3, é possível observar quais fatores sócio-demográficos e de formação esportiva influenciam para divisão dos sujeitos entre os Clusters:

Tabela 3 - Qui-Quadrado.

\begin{tabular}{ccc}
\hline Variável & Qui-quadrado & Significância (p) \\
\hline Idade & $\mathbf{1 3 , 3 2 7}$ & $\mathbf{0 , 0 2 1}$ \\
\hline Modalidade & 5,183 & 0,521 \\
Tipo de Modalidade & 0,574 & 0,449 \\
Estado & 16,164 & 0,706 \\
Região & 0,701 & 0,951 \\
Deficiência & $\mathbf{4 , 1 4 0}$ & $\mathbf{0 , 0 4 2}$ \\
Tipo de Deficiência & 0,259 & 0,611 \\
Escola & 1,359 & 0,244 \\
Sexo & 0,283 & 0,595 \\
Tempo de Prática & 26,255 & 0,123 \\
Carga Horária Semanal & 16,867 & 0,327 \\
\hline
\end{tabular}

*Significante ao nível de 0,05 (2-tailed). Fonte: Autores (2017).

A partir da análise de qui-quadrado demonstrado na Tabela 3, encontrou-se que apenas duas variáveis interferiram na divisão dos sujeitos nos dois grupos, apresentando valores significativos.

A primeira, se relaciona com a idade, que pode variar de 12 a 17 anos, esta apresenta o $\mathrm{p}=0,021$. Nesta variável, o Cluster 1, considerado o grupo mais motivado e resiliente possui a moda de idade de 15 anos, já no Cluster 2, 17 anos. Isto, provavelmente, está relacionado com a idade limite de participação neste evento esportivo, sendo assim, os jovens que participam em seu ultimo ano (aqueles que possuem 17 anos), já não possuem a motivação e níveis de resiliência como quando participavam em outras edições com idades inferiores, com uma visão diferente e estimulada por expectativas dos próximos anos. Tendo em vista tal predominância de idades nos grupos, estudiosos sugerem que à medida que os jovens atletas vão ficando mais velhos, também vão se distanciando dos motivos vinculados à competição (Terra, Hernandez \& Voser, 2009). 
Além disso, Gould et al., (1996) e Vieira (1999) afirmam que com o avançar da idade, o interesse pelo esporte e pelas práticas esportivas, tende a diminuir, pois a tendência é que outros fatores comecem a despertar o interesse destes jovens, e assim, o esporte tende a cair na escala de prioridades de vida. Andrade et al., (2006) corrobora, dizendo que atletas com menos experiência no esporte, são os mais motivados intrinsecamente, ou seja, quanto mais novos, mais motivados são os atletas.

A segunda variável que interfere, é a deficiência, podendo ser física ou visual, representada pelo $\mathrm{p}=0,042$. Neste caso, proporcionalmente falando, a predominância no Cluster 1 é de pessoas com deficiência visual (Cluster $1=37,73 \%$ I Cluster $2=20,34 \%$ ), enquanto no Cluster 2, prevalece a pessoa com deficiência física (Cluster $1=62,26 \% /$ Cluster $2=$ $79,66 \%$ ). Isto demonstra que, na pesquisa, as pessoas com deficiência visual estavam mais motivadas e resilientes, que as pessoas com deficiência física.

Em relação à resiliência, ou a capacidade de superação de pessoas com deficiência visual, Rubio (2006) afirma que através da motivação para a prática do esporte adaptado, estes sujeitos aprendem a superar os obstáculos, a cooperar com os demais companheiros, a desenvolver o auto-controle e, principalmente, a persistir diante das derrotas. Assumpção (2002) complementa, apontando que a motivação para a prática esportiva por parte das pessoas com deficiência visual possibilita o conhecimento e vivencia da superação, ou seja, de sua capacidade de resiliência.

Segundo Gil (2000), o esporte promove alguns pontos importantes na vida de uma pessoa com deficiência visual, e isso, consequentemente, lhe motiva ainda mais, seja na diminuição da limitação psicomotora (em muitos casos, característico da deficiência visual), na utilização do potencial sensório-motor, no aumento perceptível da auto-confiança, da auto-aceitação, e na promoção de autonomia e independência. Além disso, essa prática esportiva também representa a oportunidade de novas possibilidades de vida, na integração social, na exploração das potencialidades e no reconhecimento (Camargo Netto \& Gonzáles, 1996).

Para Brazuna e Castro (2001), através do esporte, o atleta encontra um sentido de vida e se sente motivado por isso, pois é notável não só os ganhos nas valências físicas, mas também no desenvolvimento de independência, melhoria da saúde mental e por fim, a construção da identidade como atleta, e não somente como uma pessoa com deficiência. Nessa perspectiva, a pessoa com deficiência consegue, a partir do esporte, superar seus limites e tem a confirmação de que pode conquistar e viver isso com prazer (Assumpção, 2002). Amirallan (1997), por sua vez, ressalta que o deficiente visual, se sente mais aceito e amado, quando supera todas as adversidades que a própria deficiência o impõe. Assim, após tantos benefícios citados pelos autores supracitados, percebe-se a extrema importância da pratica esportiva nesse contexto.

As correlações encontradas entre as dimensões da motivação e resiliência, podem ser observados na Tabela 4. Para tal análise, utilizou-se o Coeficiente de Pearson, que mede o grau de correlação entre duas variáveis. Este coeficiente varia em valores entre -1 e 1, e quanto maior for seu valor absoluto, mais forte é a relação encontrada entre as variáveis. Neste tipo de análise, o valor absoluto 1 significa uma relação perfeita, enquanto valores próximos a 0 , representam a não relação entre as variáveis.

Tabela 4 - Correlação das Dimensões (Correlação de Pearson).

\begin{tabular}{|c|c|c|c|c|c|}
\hline $\begin{array}{l}\text { Resiliência/ } \\
\text { Motivação }\end{array}$ & $\begin{array}{c}\text { Auto- } \\
\text { Suficiência }\end{array}$ & $\begin{array}{c}\text { Sentido de } \\
\text { Vida } \\
\end{array}$ & Equanimidade & Perseverança & $\begin{array}{c}\text { Singularidade } \\
\text { Existencial }\end{array}$ \\
\hline $\begin{array}{l}\text { Controle de } \\
\text { Estresse }\end{array}$ & $0,347 * *$ & 0,118 & $0,340 * *$ & $0,245^{* * *}$ & $0,217^{*}$ \\
\hline Saúde & $0,234 *$ & $0,285^{* *}$ & $0,282^{* * *}$ & $0,246 * *$ & $\mathbf{0 , 3 0 4 * *}$ \\
\hline Sociabilidade & 0,138 & $-0,018$ & 0,155 & 0,122 & 0,103 \\
\hline Competitividade & $0,262 * *$ & 0,185 & 0,183 & $0,252 * *$ & $0,241 *$ \\
\hline Estética & 0,131 & 0,124 & 0,057 & $0,260 * *$ & $0,199 *$ \\
\hline Prazer & $0,305^{* *}$ & $0,221 *$ & $0,250 * *$ & $\mathbf{0 , 3 3 6 * *}$ & $0,294 * *$ \\
\hline
\end{tabular}

*Correlação significativa no nível 0,05. ** Correlação significativa no nível 0,01. Fonte: Autores (2017). 
Conforme pode ser verificado nas correlações, embora em sua maioria fraca, é que existe associação significativa entre vários fatores de motivação e resiliência. Constata-se que:

1. Quanto maior o controle de estresse (motivação), maiores os fatores de resiliência de autossuficiência $(0,347)$, equanimidade $(0,340)$, perseverança $(0,245)$ e singularidade existencial $(0,217)$. Esse resultado indica que quanto mais o atleta expressa o desejo do sujeito de obter alívio de ansiedade, angústias, irritação e estresse, a partir da prática esportiva (controle de estresse), mais características de confiança em si mesmo e autoconhecimento de seus limites ele tem; mais são capazes de encarar os acontecimentos da vida com flexibilidade e boa aceitação; maior capacidade de seguir em frente apesar das adversidades e maior a consciência de sua singularidade.

2. Quanto maior o fator relacionado à saúde, isto é a percepção dos benefícios à saúde que o exercício físico pode trazer, maiores são todos os fatores de resiliência: autossuficiência $(0,234)$, sentido da vida $(0,285)$, equanimidade $(0,282)$, perseverança $(0,246)$ e singularidade existencial $(0,304)$.

3. Quanto maior o fator de competitividade, maior os seguintes fatores de resiliência: autossuficiência $(0,282)$, perseverança $(0,252)$ e singularidade existencial $(0,241)$.

4. Quanto maior o fator relacionado a estética, maior a perseverança $(0,260)$ e singularidade existencial $(0,199)$.

5. Quanto maior o prazer relacionado à prática esportiva, maiores são todos os fatores de resiliência: autossuficiência $(0,305)$, sentido da vida $(0,221)$, equanimidade $(0,250)$, perseverança $(0,336)$ e singularidade existencial $(0,294)$.

6. O único fator de motivação que não apresentou correlação significativa com nenhuma das demais dimensões, foi o fator "Sociabilidade", sendo necessárias futuras pesquisas para buscar entender melhor essa questão, uma vez que poderia se esperar que a criação de vínculos poderia estar associado a fatores de resiliência.

Esses resultados confirmam as indicações de várias pesquisas realizadas anteriormente com atletas e praticantes de exercício físico (Samulski \& Noce, 2002; Bodas, Lazaro \& Fernandes, 2007; Cusin \& Navarro, 2013; Almeida et al., 2015; Dantas et al., 2015), trazendo uma importante contribuição para o cenário de adolescentes com deficiência, participantes de grandes competições em nível escolar.

$\mathrm{Na}$ prática do exercício físico, o sujeito tem a possibilidade de vivenciar sensações de bem-estar em geral, isso contribui com a diminuição de aspectos como a ansiedade e a depressão, trazendo uma melhoria das funções cognitivas, autoconceito, autoestima e autoconfiança de pessoas com deficiência (Santiago, Souza \& Florindo, 2005). Sendo assim, percebe-se que os benefícios trazidos pela pratica de exercício físico, são de extrema importância para as pessoas com algum tipo de deficiência, então, torna-se necessário a inserção destes sujeitos em programas de exercício físico regular (Saito, 2007).

Por outro lado, uma característica psicológica que possivelmente auxilia atletas a superarem os fatores estressantes em decorrência ao esporte e a manter um bom desempenho durante as competições é a capacidade de resiliência, reconhecida como uma adaptação positiva em relação a vivencia de um trauma ou adversidade (Nicholls et al., 2012). O estresse proveniente de situações como uma competição, ocorre em todos os níveis competitivos, idade e até mesmo, experiência dos atletas, o que determinará o desempenho, será a forma como este sujeito responde e absorve tais fatores estressantes (Jorge, Santos \& Stefanello, 2010).

Em pesquisa com deficientes visuais em relação às motivações para prática de esportes, descobriu-se níveis significativos na motivação relacionada aos fatores prazer e controle de estresse, devido ao fato de gostar e sentir-se bem em relação a pratica do exercício físico, de acordo com Palma et al., (2017). Apesar de existirem adversidades na pratica esportiva, adolescentes tem a capacidade de buscar diferentes motivações, elas podem ser tanto pelo próprio prazer, quanto pela busca de aceitação em um grupo de amigos (Boaz, 2014). 


\section{Limitações do Estudo}

O presente estudo apresentou limitação quanto ao tamanho da amostra, visto a dificuldade em conseguir muitos respondentes, devido ao período da coleta que ocorreu durante a maior competição em nível escolar em âmbito nacional, com muitas partidas ao longo do dia e uma programação intensa.

Além disso, por ser apenas uma pesquisadora realizando a coleta e pela necessidade de, em alguns casos, realizar a pesquisa juntamente com o sujeito, devido às limitações inerentes apresentadas em cada caso, de acordo com a deficiência (necessidade de auxílio na leitura e escrita do Termo de Assentimento, anamnese e dos instrumentos, individualmente).

Sendo assim, fica evidente a necessidade de estudos semelhantes com grupos maiores e com a representação de todos os estados brasileiros que estiverem participando da competição.

\section{Conclusão}

Com os resultados encontrados, concluiu-se que os participantes da pesquisa obtiveram, de forma geral, bons e ótimos níveis de motivação e resiliência. Desta forma, foi possível definir dois tipos de perfis predominantes dentre o grupo principal, um com altos níveis de motivação e resiliência para o esporte, e o segundo, com níveis moderados das mesmas variáveis para prática esportiva.

Referente às associações entre os perfis e as características sócio-demograficas e de formação esportiva dos sujeitos, percebeu-se que somente a idade e a deficiência, possuíram interferência na divisão dos sujeitos.

Assim, percebeu-se que, quanto à idade, o Cluster 1, considerado o grupo mais motivado e resiliente, possuía moda de idade de 15 anos e o Cluster 2, de 17 anos. Ou seja, 17 anos era a idade considerada menos motivada e resiliente, isso pode ser explicado pelo fato de que esta é uma idade limite máxima para a participação neste evento. Quanto à deficiência, a predominância no Cluster 1, foi de pessoas com deficiência visual (Cluster $1=37,73 \%$ / Cluster $2=20,34 \%$ ), enquanto no Cluster 2, prevaleceu a pessoa com deficiência física (Cluster $1=62,26 \%$ / Cluster $2=79,66 \%$ ). Isto deixa claro que, pessoas com deficiência visual estavam mais motivadas e resilientes, que as pessoas com deficiência física.

Verificou-se também que o perfil psicológico dos atletas mais motivados e resilientes, apresenta uma configuração de características muito positivas, tanto para o esporte quanto para a própria vida dos adolescentes, associando fatores relacionados à motivação, com características de resiliência, que poderão contribuir para uma melhor qualidade de vida desses atletas e para o futuro pessoal e profissional deles, independentemente de se tornarem ou não atletas de alto rendimento rumo às Paralimpíadas Mundiais.

Por fim, é importante indicar a necessidade de futuras pesquisas para avaliar possíveis relações do perfil psicológico com o desempenho no esporte, com trajetórias no esporte e nas profissões em geral, dentre outras variáveis.

\section{Agradecimentos}

As autoras expressam sua gratidão à Universidade Federal de São João Del Rei (UFSJ), ao Comitê Paralímpico Brasileiro (CPB) e à Academia Paralímpica Brasileira (APB).

\section{Referências}

Almeida, P. P. (2009). Fatores motivacionais que interferem no desempenho do atleta professional de futebol (foco na autoconfiança). Monografia (Educação Física) - Universidade Federal do Rio Grande do Sul. 29 f. https://lume.ufrgs.br/handle/10183/18831

Almeida, M. P., Dutra, N. R., Castro, H. O., Leite, R. D. \& Pires, F. O. (2015). Motivos que levam estudantes universitarios de Educação Física de Belo Horizonte à pratica regular de atividade física. EFDeportes.com, Revista Digital. Buenos Aires, 20, 210. https://www.scielo.br/pdf/rbefe/v26n4/v26n4a12.pdf

Amirallan, M. L. T. M. (1997) Psicologia do excepcional. EPU. 
Andrade, C., Keller, B., Okazaki, F. H. A., Oliveira, S. \& Coelho, R. W. (2006). A influência do tempo de prática na motivação intrínseca de atletas de futebol masculino, da categoria juvenil de clubes profissionais. Revista Digital, 11(96). https://www.efdeportes.com/efd96/motivac.htm

Assumpção, E. (2002). Psicologia do esporte adaptado: Um novo desafio da psicologia. http://www.psicologia.com.pt/artigos

Balbinotti, A. A. M. \& Barbosa, M. L. L. (2006). Inventário De Motivação À Prática Regular De Atividade Física (Imprafe-54). Laboratório De Psicologia Do Esporte - Universidade Federal Do Rio Grande Do Sul.

Boaz, P. R. (2014). Motivação para prática desportiva em jovens de uma escola pública de Porto Alegre. Monografia (Educação Física) - Universidade Federal do Rio Grande do Sul. Porto Alegre. 35 f. https://lume.ufrgs.br/handle/10183/138205

Bodas, A., Lázaro, J \& Fernandes, H. (2007) Perfil Psicológico De Prestação Dos Atletas Paralímpicos Atenas 2007. Motricidade 3(3): 33-43. https://www.redalyc.org/pdf/2730/273020486005.pdf

Brown, C. J., Butt, J. \& Sarkar, M. (2019). Overcoming Performance Slumps: Psychological Resilience in Expert Cricket Batsmen. Journal of Applied Sport Psychology. 15:1-20. https://doi.org/10.1080/10413200.2018.1545709

Busto, R. M. (2013). Dimensões do esporte adaptado - Projetos da UEL. In: Busto, R. M, Fujisawa, D. S., Marquezine, M. C. Manzini, E. J. \& Tanaka, E. D. O. (2013) Esporte, reabilitação e educação física inclusiva na qualidade de vida de pessoas com deficiência. 9-18.

Camargo Netto, F., González, J. S. (1996). Desporto adaptado a portadores de deficiência: judô. UFRS.

Cavalcante, L. L. M. (2011). Motivação de adolescentes na prática de musculação em academia. 17 f. Monografia (Graduação em Educação Física) Faculdade de Educação Física. Universidade Católica de Brasília. Brasília - DF. https://docplayer.com.br/77377004-Leilane-lemos-mendanha-cavalcantemotivacao-de-adolescentes-na-pratica-de-musculacao-em-academia.html

Cevada, T., Cerqueira, L. S., Moraes, H. S., Santos, T. M., Pompeu, F. A. M. S. \& Deslandes, A. C. (2012). Relação entre esporte, resiliência, qualidade de vida e ansiedade. Reista.de Psiquiatria Clínica. 39(3). http://dx.doi.org/10.1590/S0101-60832012000300003

Codonhato, R., Vissoci, J. R. N., Junior, J. R. A. N., Mizoguchi, M. V \& Fiorese, L. (2018). Impact of resilience on stress and recovery in athletes. Revista Brasileira de Medicina do Esporte, 24(5), 352-356. http://dx.doi.org/10.1590/1517-869220182405170328

Cusin, M. A. \& Navarro, A. C. (2013). Perfil Psicológico Das Atletas Femininas Da Federação Paulista De Futsal. Revista Brasileira De Futsal E Futebol, Edição Especial: Pedagogia Do Esporte, São Paulo, 5(18), 276-283. http://www.rbff.com.br/index.php/rbff/article/view/188

Dantas, R., Paz, G. A., Straatmann, V. S. \& Lima, V. P. (2015). Fatores motivacionais de idosos praticantes do método pilates. Revista Brasileira de Promoção da Saúde, Fortaleza, 28(2): 251-256. https://periodicos.unifor.br/RBPS/article/view/3534/pdf

Dosil, J. (2004). Motivación: Motor Del Deporte. Psicologia De La Atividad Física Y Del Deporte. Mcgrawhill.

Figueiredo, N. M. A. (2009). Método e Metodologia na Pesquisa Científica. (3a ed.), Yendis Editora.

Fletcher, D. \& Sarkar, M. (2012). A grounded theory of psychological resilience in Olympic champions. Psychol Sport Exerc, 13: 669-678. https://doi.org/10.1016/j.psychsport.2012.04.007

Fonseca, J. J. S. (2002). Apostila de Metodologia da pesquisa científica. Universidade Estadual do Ceará.

Freitas, C. M. S. M, Santiago, M. S., Viana, A. T., Leão, A. C. \& Freyre, C. (2007). Aspetos Motivacionais Que Influenciam A Adesão E Manutenção De Idosos A Programas De Exercícios Físicos. Revista Brasileira De Cineantropometria E Desempenho Humano. V.9, N.1, 92-100. Acesso em: 20 de Março de 2018. https://pesquisa.bvsalud.org/portal/resource/pt/lil-454229

Garcia, R. P., Medeiros, C. \& Zaremba, R. S. (2011). Desenvolvendo Competências De Vida Através Do Esporte: Relato De Uma Experiência. Revista Brasileira De Psicologia Do Esporte. 4(1), 1-13. http://cev.org.br/biblioteca/desenvolvendo-competencias-vida-atraves-esporte-relatode-uma-experiencia/

Gil, A. C. (2008). Métodos e técnicas de pesquisa social. (6a ed.), Atlas.

Gil, M. (2000). Deficiência Visual. Brasília: MEC. Secretaria de educação à distância, 2000.

Gonçalves, C. E., Silva, M. J. C., Chatzisarantis, N., Lee, M. J. \& Cruz, J. (2006). Tradução E Validação Do Saq (Sports Attitudes Questionnaire) Para Jovens Praticantes Desportivos Portugueses Com Idades Entre 13 E Os 16 Anos. Revista Portuguesa De Ciências Do Desporto. 6(1), $39-47.10 .5628$ / rpcd.06.01.38

Gould, G., Tuffey, S., Udry, E. \& Loehr, J. (1996). Burnout in competitive junior tennis players: I. A quantitative psychological assessment. The Sport Psychologist, Champaign, v.10, p.322-40. https://psycnet.apa.org/record/1996-09105-002

Grunspun, H. (2006). Criando filhos vitoriosos: quando e como promover a resiliência. Atheneu.

Hair, J. J. F., Black, W. C., Barbin, B. J., Anderson, R. E. \& Tathan, R. L. (2005). Análise Multivariada de Dados. (5a ed.), Bookman.

Hasselmann, M. H. \& Reichenheim, M. E. (2003). Adaptação Transcultural Da Versão Em Português Da Conflict Tactics Scales Form R (Cts-1), Usada Para Aferir Violência No Casal: Equivalência Semântica E De Mensuração. Cad Saúde Pública. https://doi.org/10.1590/S0102-311X2003000400030

Herdman, M., Fox-Rushby, J. \& Badia, X. (1998). A Model Of Equivalence In The Cultural Adaptation Of Hrqol Instruments: The Universalist Approach. Qual Life Res, 7:323-35. DOI:: 10.1023 / a: 1024985930536

João, A. \& Fernandes Filho, J. (2002). Identificação Do Perfil Genético, Somatotípico E Psicológico Das Atletas Brasileiras De Ginástica Olímpica Feminina De Alta Qualificação Esportiva. Fitness \& Performance Journal. 1(2), 12-19. 10.3900/fpj.1.2.12.p 
Jorge, S. R., Santos, P. B. \& Stefanello, J. M. F. (2010). O cortisol salivar como resposta fisiológica ao estresse competitivo: uma revisão sistemática. Rev Educ Fis/UEM, 21(4): 677-686. 10.4025/reveducfis.v21i4.9053

Levermore, R. (2008). Sport a new engine of development? Progress in development studies, 8, 183-190. 10.1177/146499340700800204

Martin, J., \& Wheeler, G. (2011). Psychology. In Vanlandewijck, Y. \& Thompson, W. (Eds.), Handbook of Sports Medicine and Science - The Paralympic Athlete (pp. 116- 135). John Wiley \& Sons. Ltd.

Matos, D., Cruz, J., \& Almeida, L (2011). Excelência no desporto: Para uma compreensão da arquitectura psicológica dos atletas de elite. Motricidade, 7(4), 27-41. 10.6063/motricidade.7(4).87

Medina, M. F. \& Fernandes Filho, J. (2002). Identificação Dos Perfis Genético E Somatotípico Que Caracterizam Atletas De Voleibol Masculino Adulto De Alto Rendimento No Brasil. Fitness \& Performance Journal. 1(4), 12-19. https://docplayer.com.br/16527332-Identificacao-dos-perfis-genetico-esomatotipico-que-caracterizam-atletas-de-voleibol-masculino-adulto-de-alto-rendimento-no-brasil.html

Milham, L. (2007). Getting Back: Performance Resilience In.: Division I And Community College Athletes. Fullerton: Tese De Mestrado Apresentada À Faculdade Da Universidade Do Estado Da California.

Moraes, C. L., Hasselmann, M. H. \& Reichenheim, M. E. (2002). Adaptação Transcultural Para O Português Do Instrumento Revised Conflict Tactics Scales (Cts2), Utilizado Para Identificar Violência Entre Casais. Cad Saúde Pública. https://doi.org/10.1590/S0102-311X2002000100017

Nicholls, A. R., Morley, D. \& Perry, J. L. (2016). The model of motivational dynamics in sport: resistance to peer influence, behavioral engagement and disaffection, dispositional coping and resilience. Front Psychol, 6:1-9. 10.3389 / fpsyg.2015.02010

Palma, L. E., Feck, R. M., Patias, B. C., Londero, A. B., Bagatini, G. Z., Dorneles, M. S. \& Machado, V. F. (2017). Aspectos motivacionais para a pratica de atividade física por pessoas com deficiência visual. Revista Kinesis, 35(2), 43- 49. https://periodicos.ufsm.br/kinesis/article/view/25060

Parsons, A. G. W. (2016). O Brasil é um líder mundial no desenvolvimento do esporte paralímpico. Julho. Blog Vencer Limites Pessoas com Deficiência. Entrevista concedida a Luiz Alexandre Souza Ventura. https://brasil.estadao.com.br/blogs/vencer-limites/o-brasil-e-um-dos-lideres-mundiais-nodesenvolvimento-do-esporte-paralimpico/

Perin, P. C., Dias, C. S., Corte-Real, N. J., Andrade, A. L. \& Fonseca, A. M. (2015). Análise Fatorial Confirmatória Da Versão Brasileira Da Escala De Resiliencia (Er-Brasil). Gerais: Revista Interinstitucional De Psicologia, http://pepsic.bvsalud.org/scielo.php?script=sci_arttext\&pid=S198382202015000300007\#: :text=A\%20partir\%20de\%20procedimentos\%20de,equanimidade\%2C\%20perseveran\%C3\%A7a\%20e\%20singularidade\%20existenci al.

Pujals, C. \& Vieira, L. F. (2002). Análise Dos Fatores Psicológicos Que Interferem No Comportamento Dos Atletas De Futebol De Campo. Revista Da Educação Física/UEM. Maringá, 13(1), 89-97, 10.4025 / reveducfisv13n1p89-97

Rice, S. M., Purcell, R., Da Silva, S., Mawren, D., McGorry, P. D. \& Parker, A. G. (2016). The mental health of elite athletes: a narrative systematic review. Sports medicine, 46(9), 1333-1353, 10.1007 / s40279-016-0492-2

Rubio, K. (2006). O imaginário da derrota no esporte contemporâneo. Psicologia e Sociedade, 18(1). https://doi.org/10.1590/S0102-71822006000100012

Saito, S. P. (2007). Motivos que levam indivíduos portadores de deficiência visual a participarem de atividades desportivas adaptadas de alto nível. Revista Mineira de Educação Física, 15(2), 20-32.

Samulski, D. (2009). Psicologia Do Esporte. Editora Manole.

Samulski, D. \& Noce, F. (2002). Perfil Psicológico De Atletas Paraolímpicos Brasileiros. Rev Bras Med Esporte. 8(4). https://doi.org/10.1590/S151786922002000400005

Sanches, S. M. \& Rubio, K. (2010). Reflexões Sobre O Conhecimento De Resiliência: Adversidades No Contexto Esportivo. Revista Polemica, 9(2), 92-98, https://www.e-publicacoes.uerj.br/index.php/polemica/article/download/2752/1874

Santiago, A. L. S. P., Souza, M. T. \& Florindo, A. A. (2005). Comparação da percepção da autoimagem de pessoas portadoras de deficiência física praticantes de natação. Revista Digital, Buenos Aires, 10(89), https://www.efdeportes.com/efd89/defic.htm\#: :text=Os\%20resultados\%20 obtidos\%20na\%20compara\%C3\%A7\%C3\%A30,p\%3C0\%2C05).

Santos, R. M. P., Ribeiro, M. A. \& Carvalho, A. B. F. (2014). Perfil Psicológico De Atletas De Voleibol. Coleção Pesquisa Em Educação Física, Várzea Paulista, 13(1), 119-128.

Silva, I. P. G. (2019). Motivação e Autoestima entre atletas paralímpicos do estado da Paraíba. Monografia (Graduação em Educação Física) - Departamento de Educação Física. Universidade Federal da Paraíba. João Pessoa - PB. https://repositorio.ufpb.br/jspui/bitstream/123456789/17580/1/IPGS04022020.pdf

Silva, L. H. O. (2016). Perfil das pessoas que iniciam na prática do esporte adaptado coletivo na região metropolitana de Goiânia. Monografia de Conclusão de Curso (Graduação em Educação Física) - Universidade Federal de Goiás. https://repositorio.bc.ufg.br/handle/ri/12002

Silva, P. V. C. \& Costa Jr, A. L. (2011). Efeitos da atividade física para a saúde de crianças e adolescentes. Psicol. Argum. 29(64), 41-50 https://periodicos.pucpr.br/index.php/psicologiaargumento/article/view/19915

Soares, E. D. F. \& Machado, A. J. S. (2004). Perfil Do Atleta De Mountain Bike Da Cidade De Ipatinga-Mg. Revista Online Unileste. 2(2), 1-17. https://silo.tips/download/perfil-do-atleta-de-mountain-bike-da-cidade-de-ipatinga-mg

Solano, J. P. C., Bracher, E. S. B., Faisal-Cury, A., Ashmawi, H. A., Carmona, M. J. C., Neto, F. L. \& Vieira, J. E. (2016). Factor structure and psychometric properties of the Connor-Davidson resilience scale among Brazilian adult patients. Sao Paulo Medical Journal, 134.5: 400-406. https://doi.org/10.1590/15163180.2015 .02290512 
Research, Society and Development, v. 10, n. 4, e17310414044, 2021

(CC BY 4.0) | ISSN 2525-3409 | DOI: http://dx.doi.org/10.33448/rsd-v10i4.14044

Sylta, Ø., Tønnessen, E., Hammarström, D., Danielsen, J., Skovereng, K., Ravn, T., Rønnestad, B. R., Sandbakk, Ø. \& Seiler, S. (2016). The effect of different high-intensity periodization models on endurance adaptations. Medicine \& Science in Sports \& Exercise. 48(11):2165-74. 10.1249 / MSS.0000000000001007

Terra, G. B., Hernandez, J. A. E. \& Voser, R. C. (2009). A motivação de crianças e adolescentes para a prática do futsal. EFDeportes.com, Revista Digital. https://www.efdeportes.com/efd128/a-motivacao-de-criancas-e-adolescentes-para-a-pratica-do-futsal.htm

Veloso, P., Celani, G. \& Scheeren, R. (2018). From the generation of layouts to the production of construction documents: Anapplication in the customization of a partmentplans. Automation in Construction, 96: 224-235. https://bv.fapesp.br/en/publicacao/156003/from-the-generation-of-layouts-to-the-production-ofconstruc/

Wagnild, G. M. \& Young, H. M. (1993). Development And Psychometric Assessment Of The Resilience Scale. Journal Of Nursing Easurement, 165-178. Https://Pubmed.Ncbi.Nlm.Nih.Gov/7850498/

Wagstaff, C. R., Sarkar, M., Davidson, C. L. \& Fletcher, D. (2016). Resilience in sport: a critical review of psychological processes, sociocultural influences, andorganizational dynamics. In: The organizational psychology of sport (p. 138-168). Routledge.

Weinberg R. S., \& Gould, D. (2017). Fundamentos da Psicologia do Esporte e do Exercício. (6a ed.), Artmed. 